\title{
Comparing the Scientific Production of Peruvian Universities with Equitable Indexes
}

\author{
Avid Roman-Gonzalez ${ }^{*}, 1$, Antony Ciriaco-Susanibar ${ }^{1}$, Natalia I. Vargas-Cuentas ${ }^{2}$ \\ ${ }^{1}$ Universidad Nacional Tecnológica de Lima Sur, Aerospace Sciences and Health Research Laboratory (INCAS-Lab), 15314, Peru \\ ${ }^{2}$ Universidad de Ciencias y Humanidades, Image Processing Research Laboratory (INTI-Lab), 15834, Peru
}

\begin{tabular}{l} 
A R T I C L E I N F O \\
\hline Article history: \\
Received: 17 June, 2019 \\
Accepted: 27 July, 2019 \\
Online: 16 August, 2019 \\
\hline Keywords: \\
University ranking \\
SCOPUS \\
Published papers \\
Research measurement \\
Research
\end{tabular}

\begin{abstract}
A B S T R A C T
In recent years, research has been taking an important role in Peru. There are different sources of funding for research projects, research internships, the publication of scientific articles, scholarships for postgraduate studies, among others. This importance of research has resulted in an interest in being able to measure and compare universities according to their scientific production. One of the main factors to be used to make these comparisons is the number of articles published and indexed in SCOPUS. However, this measure is not entirely fair, since it is not equitable to compare a large university with a small university since large universities have more human resources to publish scientific articles. Seeing this reality, in the present work, we present a comparison of scientific production in Peruvian universities, taking into account the size of the institutions. Among the results obtained, we can observe a change in the ranking of small universities that invest heavily in research.
\end{abstract}

\section{Introduction}

Research is essential for the development of a country. It has already been seen during the Second World War; the main advances were made thanks to the scientists [1]. One of these advances, for example, is the development of the atomic pump thanks to the work of Albert Einstein [2].

According to what is argued by Roman-Gonzalez in [3], the research should be supported by a tripod composed of the university, the government, and the private enterprise, as shown in Figure 1.

The university is an essential support of the tripod since the university is not only called to train new professionals, but also is hoped to contribute with human knowledge, and this is achieved through research. Professors are the main actors of the investigation, but it is also the students who can collaborate in the publication of scientific articles.

The government is another significant support because it is called to define the policies and laws that promote research in a country. One of these policies that encourage research is, for example, the tax benefit for companies investing in research and technological development that currently applies in Peru [4].

\footnotetext{
*Avid Roman-Gonzalez, Universidad de Ciencias y Humanidades, Image Processing Research Laboratory (INTI-Lab), +51984904763, avid.romangonzalez@ieee.org

www.astesj.com

https://dx.doi.org/10.25046/aj0404488
}

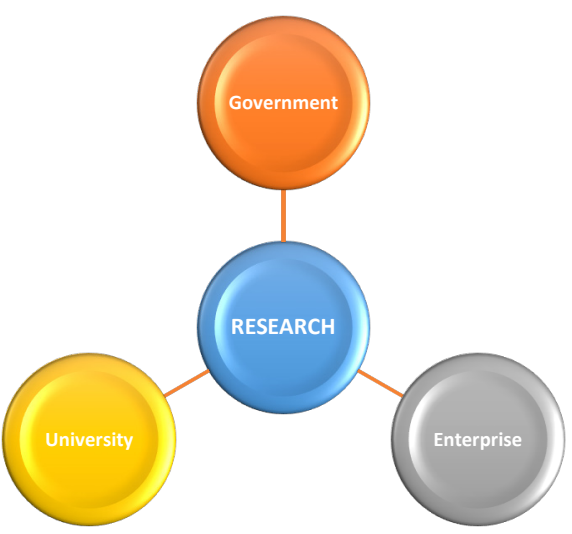

Figure 1: Tripod of the research

The private company completes the tripod since they are called to finance the research. This financing can mainly be oriented to solve problems or challenges of the same company.

In developed countries, the private sector has many challenges to solve and relies on the university (professors and students) and their capacity to face these challenges. In this way, the company finances the different research projects under a political framework established by the government.

Unfortunately, this scenario is not replicated in developing countries, mainly because companies do not trust in the 


\section{A. Roman-Gonzalez et al. / Advances in Science, Technology and Engineering Systems Journal Vol. 4, No. 4, 394-403 (2019)}

university's abilities to solve problems, and because the government does not have adequate policies to motivate the research activities.

In Peru, we are in the process of transition from one panorama (the second) to the other (the first), hoping to obtain good results.

The research is a process that follows the scientific method, and that must end in the publication of one or several scientific articles. The scientific article is nothing more than a report of the research work carried out under a pre-established format. The release of scientific articles is critical because it is necessary to let the world know about what one is researching because whatever the subject one is investigating, one is sure that on the other side of the world more research groups are working on the same subject and/or similar topics. Thanks to the publications one can get in touch with other research groups, collaborative works are achieved, joint efforts to obtain better results, scholarship opportunities, and research stay, among other benefits.

Because the research is so essential, different rankings have been established that evaluate the universities in the function of the investigation and scientific production they carry out. Among these rankings we can mention the ones elaborated by SCIMAGO [5], America Economia [6], National Superintendency of Higher University Education (SUNEDU - Superintendencia Nacional de Educacion Superior Universitaria) of Peru [7], Academic Ranking of World Universities (ARWU) [ 8], Webometrics [9], QS World University Ranking [10], among others. For the elaboration of these rankings, different parameters are used such as scientific production, scientific talent pool, citations by document, web size, international collaboration, domain's inbound links, percentage of publication in journals of the first quartile, average scientific quality, among others [3] [6] [11].

The universities carry out and implement different strategies to improve their position in the rankings. Some use new methodologies in their teaching processes as in [12], and others develop a whole program of formative research to get undergraduate students to start publishing scientific articles, as mentioned in [13].

One of the most critical rankings developed as a function of scientific production is the one produced by the SCIMAGO group [5] [11].

SCIMAGO generates two types of ranking, one is the SIR World, which is a worldwide raking in which only institutions that have publications indexed in SCOPUS equal to or higher than 100 per year are included. Peru has had a growth in the number of institutions that enter in this ranking since 2009. In Figure 2, we can see this evolution where one can see that Peru went from 1 institution in 2009, passed through 4 institutions in 2012 and reached seven institutions in 2018. In Figure 2, one can also see the evolution of the best positioned Peruvian university in the ranking.

Apart from the SIR World, there is also the SIR Iber ranking that includes only Ibero-American countries. For entering into this ranking, it is only necessary to have at least one article published and indexed in SCOPUS. In Figure 3, one can see the evolution of the number of Peruvian institutions included in the SIR Iber ranking. One can see that by 2009 there were 50 Peruvian institutions and by 2018 the number increased to 77 .

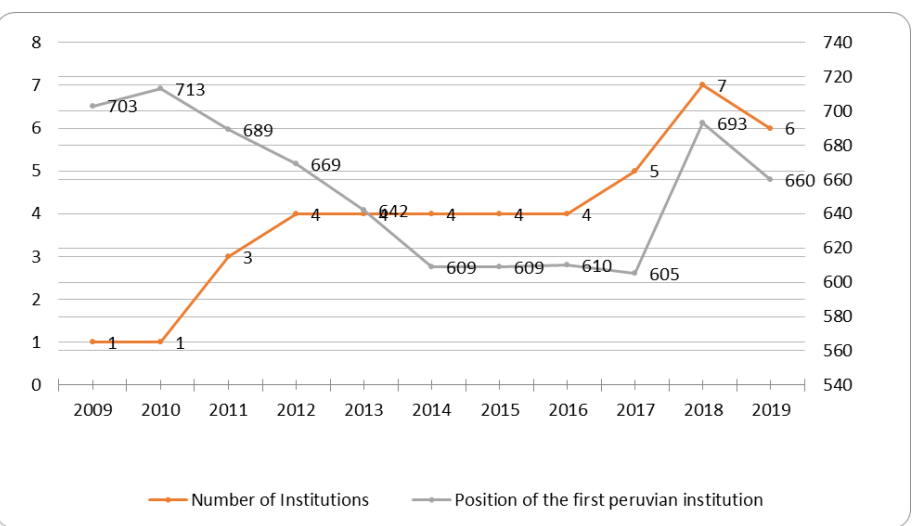

Figure 2: Evolution of the number of Peruvian institutions in the SIR World rankings [5] [11]

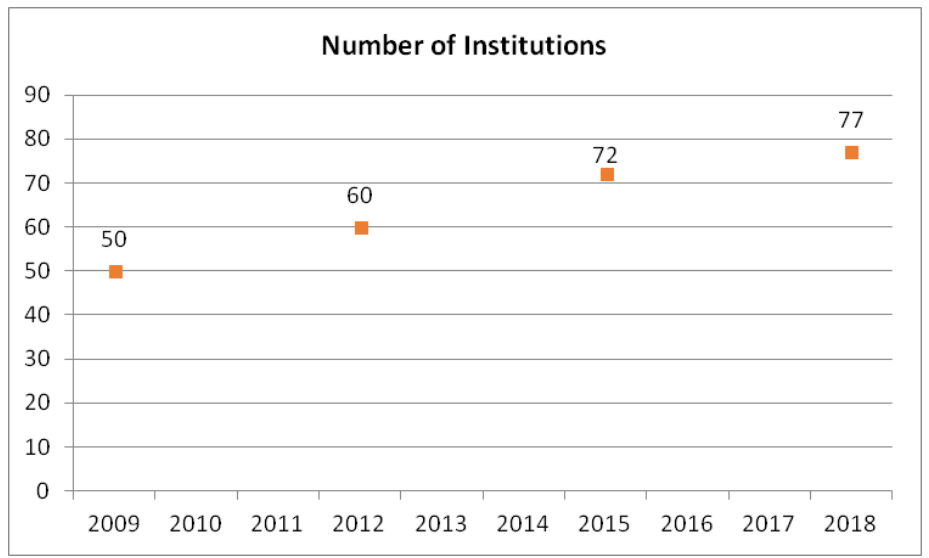

Figure 3: Evolution of the number of Peruvian institutions in the SIR Iber ranking [5] [11]

Among all the parameters mentioned above, one of the most used for the elaboration of the rankings of universities that evaluate scientific production is the number of published scientific articles. While it is true that it is an important parameter, it is not the most appropriate way to compare large universities that have many professors and students, compared to small universities with fewer professors and students. The universities with the most significant number of professors and students have more considerable human resources to carry out research and therefore, to have higher scientific production.

Faced with this situation, some proposals try to use more equitable parameters to compare scientific production in large and small universities. One of these works is the one presented in [14], where the authors use the ratio between the number of publications and the number of professors.

In this paper, one proposes other parameters that can also be used to develop rankings more equitably when comparing universities of different sizes and ages.

The continuation of this work is structured as follows: Section II shows the data source for the analysis carried out as well as the methodology followed. Section III presents the rankings obtained as a result. Finally, in Section IV, these results will be analyzed as part of the discussion and conclusions 


\section{Methodology}

In Peru, due to the new University Law 30220, the majority of universities are investing more in research. Likewise, different analyzes on scientific production are being prepared, such as the one presented in [15].

The most equitable way to compare the scientific production of universities is taking into account their size. One way to include the size of the university in the elaboration of the rankings is to use the number of students. Another idea of having reference to the size of the university is to consider the amount of professor, as was done in [14].

There is a discrepancy due to the fact of considering the number of students or the number of professors since the students are not the main ones involved in the formal research process. Professors are called to do research; however, it is debatable to take into account the total number of professors or only take the number of full-time professors. It is arguably to say that just full-time professors do research.

Faced with this situation, in the present work, one takes into account the number of professors qualified as REGINA researchers. REGINA is the National Registry of Researchers by its initials in Spanish (Registro de Investigadores en Ciencia y Tecnología del Sistema Nacional de Ciencia, Tecnología e Innovación Tecnológica). REGINA is a proposal of CONCYTEC (National Council of Science, Technology and Technological Innovation) that takes into account a set of parameters to evaluate the professionals who research to qualify them as researchers [16]. When considering the number of REGINA researchers per university, we will be sure to take into account those who are involved in scientific activity.

Likewise, in the present work, one will also consider the number of authors identified in SCOPUS to measure with an equitable way the scientific production in large and small universities.

In this section, the methodology followed for the elaboration of the proposed equitable rankings will be described.

\subsection{Data Collection}

The data analyzed in the present work correspond to the articles published by each university. For this, the SCOPUS database belonging to ELSEVIER will be taken as a reference. The publications of 2017, 2018 will be analyzed, and the total papers will also be taken into account.

The analysis of the present work will be carried out on all the Peruvian universities that have an ID in the SCOPUS database. According to the SUNEDU biennial report [17], in Peru, there are 142 universities of which ten did not provide services at the date of publication of the mentioned report, besides there are four graduate schools and the Facultad de Teología Pontificia y Civil de Lima that has university rank.
To access the SCOPUS data, CONCYTEC provides access to all the people registered in CTI Vitae (bio-sketches related to Science and Technology) through the credentials created for that purpose. In this sense, there will be direct access to SCOPUS, as shown in Figure 4. CTI Vitae is a database that allows people who carry out science, technology, and innovation (CTI) activities to register their resumes.

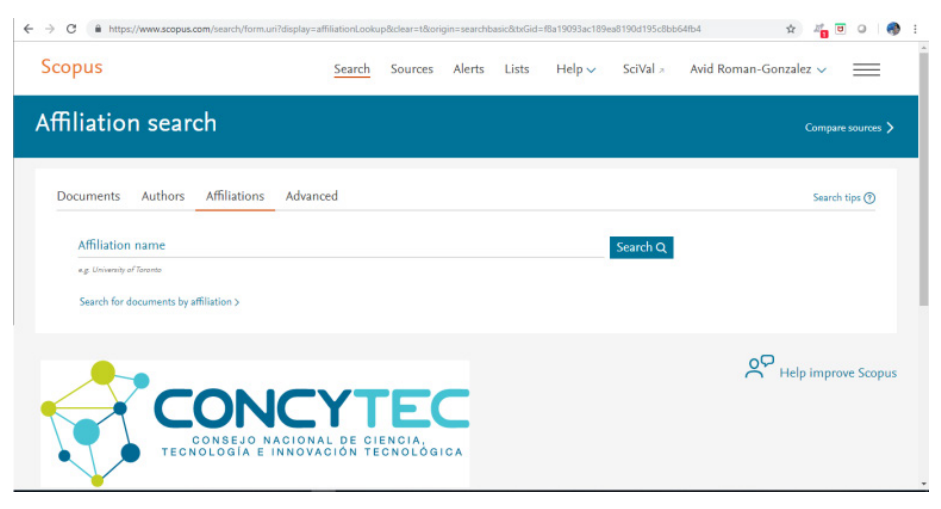

Figure 4: Access to the SCOPUS database

Likewise, the information will be collected regarding the number of professors qualified as REGINA that each university has. For this purpose, the portal created by CONCYTEC will be accessed, as shown in Figure 5

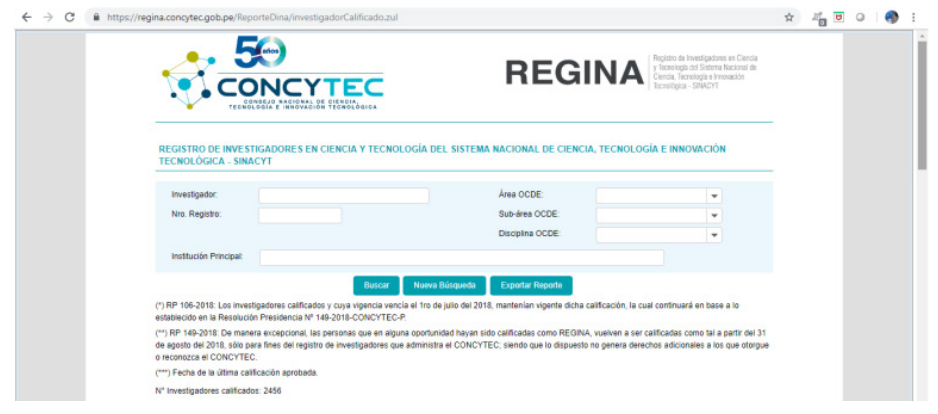

Figure 5: Access to the portal to identify the REGINA researchers for each university

All data were collected on 31st March 2019.

\subsection{The ratio between the number of publication and number of researches and authors}

For the elaboration of equitable rankings when comparing large universities with small universities, we propose to use two ratios. The first ratio will be considering the number of publications in a given year, divided by the number of REGINA researchers for each university (Equation 1). The second ratio considers the total number of publications divided by the number of authors identified in SCOPUS by each university (Equation 2).

$$
\begin{aligned}
& R_{p / r}=P / R \\
& R_{p / a}=P / A
\end{aligned}
$$

Where:

- $R_{p / r}$ is the ratio between the number of paper indexed in SCOPUS and the number of REGINA researchers that will be used to elaborate the ranking. 
A. Roman-Gonzalez et al. / Advances in Science, Technology and Engineering Systems Journal Vol. 4, No. 4, 394-403 (2019)

- $\quad R_{p / a}$ is the ratio between the number of paper indexed in SCOPUS and the number of authors identified in SCOPUS that will be used to elaborate the ranking.

- $\quad P$ is the total number of published paper indexed in SCOPUS.

- $R$ is the number of REGINA researchers for each university, according to CONCYTEC.

- $A$ is the number of total authors that publish papers with the affiliation of the university under analysis, according to SCOPUS.

\section{Results}

Of all the Peruvian universities, at the date of data collection, only 66 universities have their ID in SCOPUS, have at less one publication in 2017 and 2018, and have REGINA researchers.

After the calculation of the ratios mentioned in Section II, the following results are obtained and compared with the classical ranking based only on the number of publications.

Table I and Table II show the rankings only according to the number of paper for the years 2017 and 2018, respectively.

Table 1: Ranking based on the number of papers published in 2017

\begin{tabular}{|c|c|c|}
\hline $\mathbf{N}^{\circ}$ & University & $\begin{array}{c}\text { Papers } \\
\text { SCOPUS } \\
2017(P) \\
\end{array}$ \\
\hline 1 & Pontificia Universidad Católica del Perú & 415 \\
\hline 2 & Universidad Peruana Cayetano Heredia & 402 \\
\hline 3 & Universidad Nacional Mayor de San Marcos & 366 \\
\hline 4 & Universidad Peruana de Ciencias Aplicadas S.A.C. & 138 \\
\hline 5 & Universidad de San Martín de Porres & 101 \\
\hline 6 & Universidad Nacional de San Agustín de Arequipa & 76 \\
\hline 7 & Universidad Nacional Agraria La Molina & 76 \\
\hline 8 & Universidad Nacional de Ingeniería & 65 \\
\hline 9 & Universidad Científica del Sur S.A.C. & 64 \\
\hline 10 & Universidad Nacional de San Antonio Abad del Cusco & 54 \\
\hline 11 & Universidad Católica San Pablo & 51 \\
\hline 12 & Universidad del Pacífico & 50 \\
\hline 13 & Universidad de Piura & 46 \\
\hline 14 & Universidad Privada Antenor Orrego & 44 \\
\hline 15 & Universidad San Ignacio de Loyola S.A. & 44 \\
\hline 16 & Universidad Nacional de Trujillo & 41 \\
\hline 17 & Universidad de Ciencias y Humanidades & 39 \\
\hline 18 & Universidad Privada del Norte S.A.C. - & 36 \\
\hline 19 & Universidad Continental S.A.C. $(* 7)$ & 36 \\
\hline 20 & Universidad ESAN & 30 \\
\hline 21 & Universidad Ricardo Palma & 29 \\
\hline 22 & Universidad Nacional Federico Villarreal & 27 \\
\hline 23 & Universidad Nacional San Luis Gonzaga & 24 \\
\hline 24 & Universidad Nacional de Piura & 23 \\
\hline 25 & Universidad de Ingeniería y Tecnología & 22 \\
\hline
\end{tabular}

$\underline{\text { www.astesj.com }}$

\begin{tabular}{|c|c|c|}
\hline 26 & Universidad Católica de Santa María & 19 \\
\hline 27 & Universidad Nacional del Altiplano & 17 \\
\hline 28 & Universidad de Lima & 17 \\
\hline 29 & Universidad Nacional Pedro Ruiz Gallo & 14 \\
\hline 30 & Universidad Nacional de la Amazonía Peruana & 13 \\
\hline 31 & Universidad Católica Los Ángeles de Chimbote $(* 4)$ - & 13 \\
\hline 32 & Universidad Católica Santo Toribio de Mogrovejo - & 13 \\
\hline 33 & Universidad Nacional de San Cristóbal de Huamanga & 10 \\
\hline 34 & Universidad César Vallejo S.A.C. & 10 \\
\hline 35 & Universidad Señor de Sipán & 10 \\
\hline 36 & Universidad Nacional del Centro del Perú & 9 \\
\hline 37 & Universidad Privada San Juan Bautista S.A.C. $(* 6)$ & 9 \\
\hline 38 & Universidad Nacional Micaela Bastidas de Apurímac & 8 \\
\hline 39 & Universidad Nacional de Tumbes & 7 \\
\hline 40 & Universidad Nacional de Cajamarca & 6 \\
\hline 41 & Universidad Nacional Santiago Antúnez de Mayolo & 6 \\
\hline 42 & Universidad Privada de Tacna & 5 \\
\hline 43 & Universidad Alas Peruanas & 5 \\
\hline 44 & Universidad Privada Norbert Wiener & 4 \\
\hline 45 & Universidad Católica Sedes Sapientiae & 4 \\
\hline 46 & Universidad La Salle & 4 \\
\hline 47 & Universidad Nacional del Callao & 3 \\
\hline 48 & Universidad Nacional de Ucayali & 3 \\
\hline 49 & Universidad Nacional de Huancavelica & 3 \\
\hline 50 & $\begin{array}{l}\text { Universidad Nacional Toribio Rodríguez de Mendoza de } \\
\text { Amazonas }\end{array}$ & 3 \\
\hline 51 & Universidad Nacional José María Arguedas & 3 \\
\hline 52 & Universidad Andina Néstor Cáceres Velásquez & 3 \\
\hline 53 & Universidad Andina del Cusco & 3 \\
\hline 54 & Universidad Autónoma del Perú & 3 \\
\hline 55 & Universidad Nacional Agraria de la Selva & 2 \\
\hline 56 & Universidad Nacional Daniel Alcides Carrión & 2 \\
\hline 57 & Universidad Nacional de San Martín & 2 \\
\hline 58 & Universidad Nacional del Santa & 2 \\
\hline 59 & Universidad Nacional Intercultural de la Amazonía & 2 \\
\hline 60 & Universidad Nacional Autónoma de Chota & 2 \\
\hline 61 & Universidad Peruana Los Andes & 2 \\
\hline 62 & Universidad Peruana Unión & 2 \\
\hline 63 & Universidad Tecnológica del Perú & 2 \\
\hline 64 & Universidad Nacional Jorge Basadre Grohmann & 1 \\
\hline
\end{tabular}

Table 2: Ranking based on the number of papers published in 2018

\begin{tabular}{|c|l|c|}
\hline $\mathbf{N}^{\circ}$ & \multicolumn{1}{|c|}{ University } & $\begin{array}{c}\text { Papers } \\
\text { SCOPUS } \\
\mathbf{2 0 1 8}(\boldsymbol{P})\end{array}$ \\
\hline 1 & Pontificia Universidad Católica del Perú & 456 \\
\hline 2 & Universidad Nacional Mayor de San Marcos & 427 \\
\hline
\end{tabular}


A. Roman-Gonzalez et al. / Advances in Science, Technology and Engineering Systems Journal Vol. 4, No. 4, 394-403 (2019)

\begin{tabular}{|c|c|c|}
\hline 3 & Universidad Peruana Cayetano Heredia & 411 \\
\hline 4 & Universidad Peruana de Ciencias Aplicadas S.A.C. & 221 \\
\hline 5 & Universidad de San Martín de Porres & 126 \\
\hline 6 & Universidad Nacional de Ingeniería & 116 \\
\hline 7 & Universidad Nacional de San Agustín de Arequipa & 115 \\
\hline 8 & Universidad Científica del Sur S.A.C. & 101 \\
\hline 9 & Universidad de Ciencias y Humanidades & 86 \\
\hline 10 & Universidad Nacional Agraria La Molina & 83 \\
\hline 11 & Universidad Nacional de San Antonio Abad del Cusco & 74 \\
\hline 12 & Universidad del Pacífico & 66 \\
\hline 13 & Universidad San Ignacio de Loyola S.A. & 63 \\
\hline 14 & Universidad Nacional de Trujillo & 53 \\
\hline 15 & Universidad Continental S.A.C. $(* 7)$ & 51 \\
\hline 16 & Universidad Privada del Norte S.A.C. - & 47 \\
\hline 17 & Universidad de Piura & 41 \\
\hline 18 & Universidad Católica San Pablo & 34 \\
\hline 19 & Universidad Privada Antenor Orrego & 31 \\
\hline 20 & Universidad de Ingeniería y Tecnología & 31 \\
\hline 21 & Universidad Nacional Federico Villarreal & 27 \\
\hline 22 & Universidad de Lima & 27 \\
\hline 23 & Universidad Nacional de la Amazonía Peruana & 26 \\
\hline 24 & Universidad ESAN & 26 \\
\hline 25 & Universidad Nacional Pedro Ruiz Gallo & 20 \\
\hline 26 & Universidad Nacional del Centro del Perú & 19 \\
\hline 27 & Universidad Nacional del Altiplano & 19 \\
\hline 28 & Universidad Católica de Santa María & 18 \\
\hline 29 & Universidad Nacional de Piura & 17 \\
\hline 30 & Universidad César Vallejo S.A.C. & 17 \\
\hline 31 & Universidad La Salle & 16 \\
\hline 32 & Universidad Nacional San Luis Gonzaga & 14 \\
\hline 33 & Universidad Privada San Juan Bautista S.A.C. (*6) & 13 \\
\hline 34 & Universidad Nacional de San Cristóbal de Huamanga & 10 \\
\hline 35 & Universidad Peruana Unión & 9 \\
\hline 36 & Universidad Católica Los Ángeles de Chimbote (*4) - & 9 \\
\hline 37 & Universidad Alas Peruanas & 9 \\
\hline 38 & Universidad Nacional Agraria de la Selva & 8 \\
\hline 39 & Universidad Nacional del Santa & 8 \\
\hline 40 & Universidad Nacional Santiago Antúnez de Mayolo & 7 \\
\hline 41 & Universidad Nacional de Ucayali & 7 \\
\hline 42 & Universidad Nacional Micaela Bastidas de Apurímac & 7 \\
\hline 43 & Universidad Privada de Tacna & 7 \\
\hline 44 & Universidad Nacional de Cajamarca & 6 \\
\hline 45 & $\begin{array}{l}\text { Universidad Nacional Toribio Rodríguez de Mendoza } \\
\text { de Amazonas }\end{array}$ & 6 \\
\hline 46 & Universidad Católica Santo Toribio de Mogrovejo - & 6 \\
\hline
\end{tabular}

\begin{tabular}{|c|l|c|}
\hline 47 & Universidad Señor de Sipán & 6 \\
\hline 48 & Universidad Nacional Jorge Basadre Grohmann & 5 \\
\hline 49 & Universidad Peruana Los Andes & 5 \\
\hline 50 & Universidad Autónoma del Perú & 5 \\
\hline 51 & Universidad Nacional del Callao & 4 \\
\hline 52 & Universidad Nacional de Huancavelica & 4 \\
\hline 53 & Universidad Nacional Intercultural de la Amazonía & 4 \\
\hline 54 & Universidad Ricardo Palma & 4 \\
\hline 55 & Universidad Privada Norbert Wiener & 4 \\
\hline 56 & Universidad Tecnológica del Perú & 4 \\
\hline 57 & Universidad Nacional de San Martín & 3 \\
\hline 58 & Universidad Nacional José María Arguedas & 3 \\
\hline 59 & Universidad Andina Néstor Cáceres Velásquez & 3 \\
\hline 60 & Universidad Andina del Cusco & 3 \\
\hline 61 & Universidad Católica Sedes Sapientiae & 2 \\
\hline 62 & Universidad Nacional Daniel Alcides Carrión & 1 \\
\hline 63 & Universidad Nacional Autónoma de Chota & 3 \\
\hline 64 & Universidad Nacional de Tumbes & 2 \\
\hline 65 & Universidad Nacional Tecnológica de Lima Sur $(* 1)$ & 3 \\
\hline 66 & Universidad Antonio Ruiz de Montoya & 2 \\
\hline
\end{tabular}

As indicated, these rankings are not equitable with small universities. In this sense, in Table III and Table IV, we can observe new classifications based on the $R_{p / r}$ ratio described in Section II for the years 2017 and 2018, respectively.

Table 3: Ranking using the ratio $R p / r$ for the year 2017

\begin{tabular}{|c|l|c|c|c|}
\hline $\mathbf{N}^{\circ}$ & \multicolumn{1}{|c|}{ University } & $\begin{array}{c}\text { REGINA } \\
\text { Researchers } \\
(\boldsymbol{R})\end{array}$ & $\begin{array}{c}\text { Papers } \\
\text { SCOPUS } \\
\mathbf{2 0 1 7}(\boldsymbol{P})\end{array}$ & $\begin{array}{c}\text { Paper } \\
\mathbf{2 0 1 7} / \\
\text { REGINA } \\
(\boldsymbol{R} \boldsymbol{p} / \boldsymbol{r})\end{array}$ \\
\hline 1 & $\begin{array}{l}\text { Universidad Nacional Federico } \\
\text { Villarreal }\end{array}$ & 1 & 27 & 27,00 \\
\hline 2 & Universidad Señor de Sipán & 1 & 10 & 10,00 \\
\hline 3 & $\begin{array}{l}\text { Universidad Continental S.A.C. } \\
(* 7)\end{array}$ & 5 & 36 & 7,20 \\
\hline 4 & $\begin{array}{l}\text { Universidad Católica Los } \\
\text { Ángeles de Chimbote }(* 4)-\end{array}$ & 2 & 13 & 6,50 \\
\hline 5 & $\begin{array}{l}\text { Universidad Nacional San Luis } \\
\text { Gonzaga }\end{array}$ & 4 & 24 & 6,00 \\
\hline 6 & $\begin{array}{l}\text { Universidad Peruana de } \\
\text { Ciencias Aplicadas S.A.C. }\end{array}$ & 23 & 138 & 6,00 \\
\hline 7 & $\begin{array}{l}\text { Universidad Privada del Norte } \\
\text { S.A.C. - }\end{array}$ & 8 & 36 & 4,50 \\
\hline 8 & $\begin{array}{l}\text { Universidad Católica Santo } \\
\text { Toribio de Mogrovejo - }\end{array}$ & 3 & 13 & 4,33 \\
\hline 9 & $\begin{array}{l}\text { Pontificia Universidad Católica } \\
\text { del Perú }\end{array}$ & 98 & 415 & 4,23 \\
\hline 10 & Universidad La Salle & 1 & 4 & 4,00 \\
\hline 11 & $\begin{array}{l}\text { Universidad Peruana Cayetano } \\
\text { Heredia }\end{array}$ & 104 & 402 & 3,87 \\
\hline 12 & $\begin{array}{l}\text { Universidad Privada Antenor } \\
\text { Orrego }\end{array}$ & 12 & 44 & 3,67 \\
\hline 13 & Universidad ESAN & 9 & 30 & 3,33 \\
\hline 14 & $\begin{array}{l}\text { Universidad de Ciencias y } \\
\text { Humanidades }\end{array}$ & 13 & 39 & 3,00 \\
\hline
\end{tabular}


A. Roman-Gonzalez et al. / Advances in Science, Technology and Engineering Systems Journal Vol. 4, No. 4, 394-403 (2019)

\begin{tabular}{|c|c|c|c|c|}
\hline 15 & $\begin{array}{l}\text { Universidad Científica del Sur } \\
\text { S.A.C. }\end{array}$ & 23 & 64 & 2,78 \\
\hline 16 & $\begin{array}{l}\text { Universidad de San Martín de } \\
\text { Porres }\end{array}$ & 42 & 101 & 2,40 \\
\hline 17 & Universidad Nacional de Piura & 10 & 23 & 2,30 \\
\hline 18 & Universidad Ricardo Palma & 13 & 29 & 2,23 \\
\hline 19 & $\begin{array}{l}\text { Universidad Nacional de San } \\
\text { Antonio Abad del Cusco }\end{array}$ & 27 & 54 & 2,00 \\
\hline 20 & $\begin{array}{l}\text { Universidad Nacional Daniel } \\
\text { Alcides Carrión }\end{array}$ & 1 & 2 & 2,00 \\
\hline 21 & Universidad del Pacífico & 25 & 50 & 2,00 \\
\hline 22 & $\begin{array}{l}\text { Universidad Católica Sedes } \\
\text { Sapientiae }\end{array}$ & 2 & 4 & 2,00 \\
\hline 23 & $\begin{array}{l}\text { Universidad de Ingeniería y } \\
\text { Tecnología }\end{array}$ & 12 & 22 & 1,83 \\
\hline 24 & $\begin{array}{l}\text { Universidad Nacional Pedro } \\
\text { Ruiz Gallo }\end{array}$ & 8 & 14 & 1,75 \\
\hline 25 & $\begin{array}{l}\text { Universidad Nacional de San } \\
\text { Cristóbal de Huamanga }\end{array}$ & 6 & 10 & 1,67 \\
\hline 26 & Universidad Privada de Tacna & 3 & 5 & 1,67 \\
\hline 27 & Universidad Católica San Pablo & 32 & 51 & 1,59 \\
\hline 28 & $\begin{array}{l}\text { Universidad Andina Néstor } \\
\text { Cáceres Velásquez }\end{array}$ & 2 & 3 & 1,50 \\
\hline 29 & Universidad de Piura & 34 & 46 & 1,35 \\
\hline 30 & $\begin{array}{l}\text { Universidad Privada San Juan } \\
\text { Bautista S.A.C. }(* 6)\end{array}$ & 7 & 9 & 1,29 \\
\hline 31 & $\begin{array}{l}\text { Universidad San Ignacio de } \\
\text { Loyola S.A. }\end{array}$ & 35 & 44 & 1,26 \\
\hline 32 & $\begin{array}{l}\text { Universidad Nacional Mayor } \\
\text { de San Marcos }\end{array}$ & 303 & 366 & 1,21 \\
\hline 33 & $\begin{array}{l}\text { Universidad Nacional de San } \\
\text { Agustín de Arequipa }\end{array}$ & 69 & 76 & 1,10 \\
\hline 34 & $\begin{array}{l}\text { Universidad Nacional de } \\
\text { Ucayali }\end{array}$ & 3 & 3 & 1,00 \\
\hline 35 & $\begin{array}{l}\text { Universidad Peruana Los } \\
\text { Andes }\end{array}$ & 2 & 2 & 1,00 \\
\hline 36 & Universidad Andina del Cusco & 3 & 3 & 1,00 \\
\hline 37 & $\begin{array}{l}\text { Universidad Privada Norbert } \\
\text { Wiener }\end{array}$ & 4 & 4 & 1,00 \\
\hline 38 & $\begin{array}{l}\text { Universidad Nacional Micaela } \\
\text { Bastidas de Apurímac }\end{array}$ & 9 & 8 & 0,89 \\
\hline 39 & $\begin{array}{l}\text { Universidad Nacional Agraria } \\
\text { La Molina }\end{array}$ & 88 & 76 & 0,86 \\
\hline 40 & Universidad de Lima & 20 & 17 & 0,85 \\
\hline 41 & $\begin{array}{l}\text { Universidad Nacional de } \\
\text { Ingeniería }\end{array}$ & 80 & 65 & 0,81 \\
\hline 42 & $\begin{array}{l}\text { Universidad Nacional de } \\
\text { Tumbes }\end{array}$ & 9 & 7 & 0,78 \\
\hline 43 & $\begin{array}{l}\text { Universidad Nacional Santiago } \\
\text { Antúnez de Mayolo }\end{array}$ & 8 & 6 & 0,75 \\
\hline 44 & $\begin{array}{l}\text { Universidad Nacional José } \\
\text { María Arguedas }\end{array}$ & 4 & 3 & 0,75 \\
\hline 45 & $\begin{array}{l}\text { Universidad Católica de Santa } \\
\text { María }\end{array}$ & 27 & 19 & 0,70 \\
\hline 46 & $\begin{array}{l}\text { Universidad Nacional de } \\
\text { Cajamarca }\end{array}$ & 9 & 6 & 0,67 \\
\hline 47 & $\begin{array}{l}\text { Universidad Nacional } \\
\text { Intercultural de la Amazonía }\end{array}$ & 3 & 2 & 0,67 \\
\hline 48 & $\begin{array}{l}\text { Universidad Nacional } \\
\text { Autónoma de Chota }\end{array}$ & 3 & 2 & 0,67 \\
\hline 49 & Universidad Alas Peruanas & 8 & 5 & 0,63 \\
\hline 50 & $\begin{array}{l}\text { Universidad Nacional del } \\
\text { Centro del Perú }\end{array}$ & 15 & 9 & 0,60 \\
\hline 51 & $\begin{array}{l}\text { Universidad Nacional de } \\
\text { Huancavelica }\end{array}$ & 5 & 3 & 0,60 \\
\hline 52 & $\begin{array}{l}\text { Universidad Autónoma del } \\
\text { Perú }\end{array}$ & 5 & 3 & 0,60 \\
\hline
\end{tabular}

\begin{tabular}{|c|l|c|c|c|}
\hline 53 & $\begin{array}{l}\text { Universidad Nacional de la } \\
\text { Amazonía Peruana }\end{array}$ & 22 & 13 & 0,59 \\
\hline 54 & $\begin{array}{l}\text { Universidad Nacional de } \\
\text { Trujillo }\end{array}$ & 70 & 41 & 0,59 \\
\hline 55 & $\begin{array}{l}\text { Universidad Nacional del } \\
\text { Callao }\end{array}$ & 6 & 3 & 0,50 \\
\hline 56 & $\begin{array}{l}\text { Universidad Nacional de San } \\
\text { Martín }\end{array}$ & 5 & 2 & 0,40 \\
\hline 57 & $\begin{array}{l}\text { Universidad Nacional del } \\
\text { Altiplano }\end{array}$ & 44 & 17 & 0,39 \\
\hline 58 & $\begin{array}{l}\text { Universidad César Vallejo } \\
\text { S.A.C. }\end{array}$ & 29 & 10 & 0,34 \\
\hline 59 & Universidad Nacional del Santa & 7 & 2 & 0,29 \\
\hline 60 & Universidad Peruana Unión & 8 & 2 & 0,25 \\
\hline 61 & $\begin{array}{l}\text { Universidad Tecnológica del } \\
\text { Perú }\end{array}$ & 9 & 2 & 0,22 \\
\hline 62 & $\begin{array}{l}\text { Universidad Nacional Agraria } \\
\text { de la Selva }\end{array}$ & 10 & 2 & 0,20 \\
\hline & $\begin{array}{l}\text { Universidad Nacional Toribio } \\
\text { Rodríguez de Mendoza de } \\
\text { Amazonas }\end{array}$ & & & \\
\hline 63 & $\begin{array}{l}\text { Universidad Nacional Jorge } \\
\text { Basadre Grohmann }\end{array}$ & 7 & 1 & 0,14 \\
\hline
\end{tabular}

Table 4: Ranking using the ratio $R p / r$ for the year 2018

\begin{tabular}{|c|c|c|c|c|}
\hline $\mathbf{N}^{\circ}$ & University & $\begin{array}{c}\text { REGINA } \\
\text { Researchers } \\
(R)\end{array}$ & $\begin{array}{l}\text { Papers } \\
\text { SCOPUS } \\
2018(P)\end{array}$ & $\begin{array}{c}\text { Paper } \\
2018 / \\
\text { REGINA } \\
(R p / r)\end{array}$ \\
\hline 1 & $\begin{array}{l}\text { Universidad Nacional Federico } \\
\text { Villarreal }\end{array}$ & 1 & 27 & 27,00 \\
\hline 2 & Universidad La Salle & 1 & 16 & 16,00 \\
\hline 3 & $\begin{array}{l}\text { Universidad Continental S.A.C. } \\
(* 7)\end{array}$ & 5 & 51 & 10,20 \\
\hline 4 & $\begin{array}{l}\text { Universidad Peruana de } \\
\text { Ciencias Aplicadas S.A.C. }\end{array}$ & 23 & 221 & 9,61 \\
\hline 5 & $\begin{array}{l}\text { Universidad de Ciencias y } \\
\text { Humanidades }\end{array}$ & 13 & 86 & 6,62 \\
\hline 6 & Universidad Señor de Sipán & 1 & 6 & 6,00 \\
\hline 7 & $\begin{array}{l}\text { Universidad Privada del Norte } \\
\text { S.A.C. - }\end{array}$ & 8 & 47 & 5,88 \\
\hline 8 & $\begin{array}{l}\text { Pontificia Universidad Católica } \\
\text { del Perú }\end{array}$ & 98 & 456 & 4,65 \\
\hline 9 & $\begin{array}{l}\text { Universidad Católica Los } \\
\text { Ángeles de Chimbote }(* 4) \text { - }\end{array}$ & 2 & 9 & 4,50 \\
\hline 10 & $\begin{array}{l}\text { Universidad Científica del Sur } \\
\text { S.A.C. }\end{array}$ & 23 & 101 & 4,39 \\
\hline 11 & $\begin{array}{l}\text { Universidad Peruana Cayetano } \\
\text { Heredia }\end{array}$ & 104 & 411 & 3,95 \\
\hline 12 & $\begin{array}{l}\text { Universidad Nacional San Luis } \\
\text { Gonzaga }\end{array}$ & 4 & 14 & 3,50 \\
\hline 13 & $\begin{array}{l}\text { Universidad de San Martín de } \\
\text { Porres }\end{array}$ & 42 & 126 & 3,00 \\
\hline 14 & Universidad ESAN & 9 & 26 & 2,89 \\
\hline 15 & $\begin{array}{l}\text { Universidad Nacional de San } \\
\text { Antonio Abad del Cusco }\end{array}$ & 27 & 74 & 2,74 \\
\hline 16 & Universidad del Pacífico & 25 & 66 & 2,64 \\
\hline 17 & $\begin{array}{l}\text { Universidad Privada Antenor } \\
\text { Orrego }\end{array}$ & 12 & 31 & 2,58 \\
\hline 18 & $\begin{array}{l}\text { Universidad de Ingeniería y } \\
\text { Tecnología }\end{array}$ & 12 & 31 & 2,58 \\
\hline 19 & $\begin{array}{l}\text { Universidad Nacional Pedro } \\
\text { Ruiz Gallo }\end{array}$ & 8 & 20 & 2,50 \\
\hline 20 & $\begin{array}{l}\text { Universidad Peruana Los } \\
\text { Andes }\end{array}$ & 2 & 5 & 2,50 \\
\hline 21 & $\begin{array}{l}\text { Universidad Nacional de } \\
\text { Ucayali }\end{array}$ & 3 & 7 & 2,33 \\
\hline 22 & Universidad Privada de Tacna & 3 & 7 & 2,33 \\
\hline
\end{tabular}


A. Roman-Gonzalez et al. / Advances in Science, Technology and Engineering Systems Journal Vol. 4, No. 4, 394-403 (2019)

\begin{tabular}{|c|c|c|c|c|}
\hline 23 & $\begin{array}{l}\text { Universidad Nacional Daniel } \\
\text { Alcides Carrión }\end{array}$ & 1 & 2 & 2,00 \\
\hline 24 & $\begin{array}{l}\text { Universidad Católica Santo } \\
\text { Toribio de Mogrovejo - }\end{array}$ & 3 & 6 & 2,00 \\
\hline 25 & $\begin{array}{l}\text { Universidad Privada San Juan } \\
\text { Bautista S.A.C. }(* 6)\end{array}$ & 7 & 13 & 1,86 \\
\hline 26 & $\begin{array}{l}\text { Universidad San Ignacio de } \\
\text { Loyola S.A. }\end{array}$ & 35 & 63 & 1,80 \\
\hline 27 & Universidad Nacional de Piura & 10 & 17 & 1,70 \\
\hline 28 & $\begin{array}{l}\text { Universidad Nacional de San } \\
\text { Cristóbal de Huamanga }\end{array}$ & 6 & 10 & 1,67 \\
\hline 29 & $\begin{array}{l}\text { Universidad Nacional de San } \\
\text { Agustín de Arequipa }\end{array}$ & 69 & 115 & 1,67 \\
\hline 30 & $\begin{array}{l}\text { Universidad Andina Néstor } \\
\text { Cáceres Velásquez }\end{array}$ & 2 & 3 & 1,50 \\
\hline 31 & $\begin{array}{l}\text { Universidad Católica Sedes } \\
\text { Sapientiae }\end{array}$ & 2 & 3 & 1,50 \\
\hline 32 & $\begin{array}{l}\text { Universidad Nacional de } \\
\text { Ingeniería }\end{array}$ & 80 & 116 & 1,45 \\
\hline 33 & $\begin{array}{l}\text { Universidad Nacional Mayor } \\
\text { de San Marcos }\end{array}$ & 303 & 427 & 1,41 \\
\hline 34 & Universidad de Lima & 20 & 27 & 1,35 \\
\hline 35 & $\begin{array}{l}\text { Universidad Nacional } \\
\text { Intercultural de la Amazonía }\end{array}$ & 3 & 4 & 1,33 \\
\hline 36 & $\begin{array}{l}\text { Universidad Nacional del } \\
\text { Centro del Perú }\end{array}$ & 15 & 19 & 1,27 \\
\hline 37 & Universidad de Piura & 34 & 41 & 1,21 \\
\hline 38 & $\begin{array}{l}\text { Universidad Nacional de la } \\
\text { Amazonía Peruana }\end{array}$ & 22 & 26 & 1,18 \\
\hline 39 & Universidad Nacional del Santa & 7 & 8 & 1,14 \\
\hline 40 & Universidad Peruana Unión & 8 & 9 & 1,13 \\
\hline 41 & Universidad Alas Peruanas & 8 & 9 & 1,13 \\
\hline 42 & Universidad Católica San Pablo & 32 & 34 & 1,06 \\
\hline 43 & Universidad Andina del Cusco & 3 & 3 & 1,00 \\
\hline 44 & $\begin{array}{l}\text { Universidad Privada Norbert } \\
\text { Wiener }\end{array}$ & 4 & 4 & 1,00 \\
\hline 45 & $\begin{array}{l}\text { Universidad Antonio Ruiz de } \\
\text { Montoya }\end{array}$ & 1 & 1 & 1,00 \\
\hline 46 & $\begin{array}{l}\text { Universidad Autónoma del } \\
\text { Perú }\end{array}$ & 5 & 5 & 1.00 \\
\hline 47 & $\begin{array}{l}\text { Universidad Nacional Agraria } \\
\text { La Molina }\end{array}$ & 88 & 83 & 0,94 \\
\hline 48 & $\begin{array}{l}\text { Universidad Nacional Santiago } \\
\text { Antúnez de Mayolo }\end{array}$ & 8 & 7 & 0,88 \\
\hline 49 & $\begin{array}{l}\text { Universidad Nacional Agraria } \\
\text { de la Selva }\end{array}$ & 10 & 8 & 0,80 \\
\hline 50 & $\begin{array}{l}\text { Universidad Nacional de } \\
\text { Huancavelica }\end{array}$ & 5 & 4 & 0,80 \\
\hline 51 & $\begin{array}{l}\text { Universidad Nacional Micaela } \\
\text { Bastidas de Apurímac }\end{array}$ & 9 & 7 & 0,78 \\
\hline 52 & $\begin{array}{l}\text { Universidad Nacional de } \\
\text { Truiillo }\end{array}$ & 70 & 53 & 0,76 \\
\hline 53 & $\begin{array}{l}\text { Universidad Nacional José } \\
\text { María Arguedas }\end{array}$ & 4 & 3 & 0,75 \\
\hline 54 & $\begin{array}{l}\text { Universidad Nacional Jorge } \\
\text { Basadre Grohmann }\end{array}$ & 7 & 5 & 0,71 \\
\hline 55 & $\begin{array}{l}\text { Universidad Nacional de } \\
\text { Cajamarca }\end{array}$ & 9 & 6 & 0,67 \\
\hline 56 & $\begin{array}{l}\text { Universidad Nacional del } \\
\text { Callao }\end{array}$ & 6 & 4 & 0,67 \\
\hline 57 & $\begin{array}{l}\text { Universidad Nacional } \\
\text { Autónoma de Chota }\end{array}$ & 3 & 2 & 0,67 \\
\hline 58 & $\begin{array}{l}\text { Universidad Católica de Santa } \\
\text { María }\end{array}$ & 27 & 18 & 0.67 \\
\hline 59 & $\begin{array}{l}\text { Universidad Nacional de San } \\
\text { Martín }\end{array}$ & 5 & 3 & 0,60 \\
\hline
\end{tabular}

\begin{tabular}{|c|l|c|c|c|}
\hline 60 & $\begin{array}{l}\text { Universidad César Vallejo } \\
\text { S.A.C. }\end{array}$ & 29 & 17 & 0,59 \\
\hline 61 & $\begin{array}{l}\text { Universidad Tecnológica del } \\
\text { Perú }\end{array}$ & 9 & 4 & 0,44 \\
\hline 62 & $\begin{array}{l}\text { Universidad Nacional del } \\
\text { Altiplano }\end{array}$ & & 19 & 0,43 \\
\hline 63 & $\begin{array}{l}\text { Universidad Nacional Toribio } \\
\text { Rodríguez de Mendoza de } \\
\text { Amazonas }\end{array}$ & 18 & 6 & 0,33 \\
\hline 64 & Universidad Ricardo Palma & 13 & 4 & 0,31 \\
\hline 65 & $\begin{array}{l}\text { Universidad Nacional } \\
\text { Tecnológica de Lima Sur }(* 1)\end{array}$ & 8 & 1 & 0,13 \\
\hline 66 & $\begin{array}{l}\text { Universidad Nacional de } \\
\text { Tumbes }\end{array}$ & 9 & 1 & 0,11 \\
\hline
\end{tabular}

Likewise, in Table $\mathrm{V}$, one can see the ranking developed using the $R_{p / a}$ ratio also described in the previous section.

Table 5: Ranking using the $R_{P / A}$ ratio

\begin{tabular}{|c|c|c|c|c|}
\hline $\mathbf{N}^{\circ}$ & University & $\begin{array}{c}\text { Papers } \\
\text { SCOPUS } \\
(P)\end{array}$ & \begin{tabular}{|l|} 
Authors \\
SCOPUS \\
$(A)$
\end{tabular} & $\begin{array}{c}\text { Paper / } \\
\text { Authors } \\
(\text { Rp/a })\end{array}$ \\
\hline 1 & $\begin{array}{l}\text { Universidad Católica Los Ángeles } \\
\text { de Chimbote }(* 4) \text { - }\end{array}$ & 35 & 10 & 3,50 \\
\hline 2 & $\begin{array}{l}\text { Universidad Nacional Tecnológica } \\
\text { de Lima Sur }(* 1)\end{array}$ & 3 & 1 & 3,00 \\
\hline 3 & $\begin{array}{l}\text { Universidad Privada del Norte } \\
\text { S.A.C. - }\end{array}$ & 111 & 37 & 3,00 \\
\hline 4 & Universidad La Salle & 39 & 13 & 3,00 \\
\hline 5 & $\begin{array}{l}\text { Universidad Científica del Sur } \\
\text { S.A.C. }\end{array}$ & 430 & 154 & 2,79 \\
\hline 6 & $\begin{array}{l}\text { Universidad San Ignacio de Loyola } \\
\text { S.A. }\end{array}$ & 181 & 76 & 2,38 \\
\hline 7 & $\begin{array}{l}\text { Universidad Peruana Cayetano } \\
\text { Heredia }\end{array}$ & 4742 & 2088 & 2,27 \\
\hline 8 & $\begin{array}{l}\text { Universidad Nacional José María } \\
\text { Arguedas }\end{array}$ & 9 & 4 & 2,25 \\
\hline 9 & Universidad del Pacífico & 267 & 128 & 2,09 \\
\hline 10 & Universidad ESAN & 200 & 98 & 2,04 \\
\hline 11 & $\begin{array}{l}\text { Universidad Continental S.A.C. } \\
(* 7)\end{array}$ & 116 & 59 & 1,97 \\
\hline 12 & $\begin{array}{l}\text { Pontificia Universidad Católica del } \\
\text { Perú }\end{array}$ & 2899 & 1515 & 1,91 \\
\hline 13 & $\begin{array}{l}\text { Universidad de Ciencias y } \\
\text { Humanidades }\end{array}$ & 170 & 90 & 1,89 \\
\hline 14 & $\begin{array}{l}\text { Universidad de San Martín de } \\
\text { Porres }\end{array}$ & 558 & 310 & 1,80 \\
\hline 15 & $\begin{array}{l}\text { Universidad Nacional Micaela } \\
\text { Bastidas de Apurímac }\end{array}$ & 32 & 18 & 1,78 \\
\hline 16 & $\begin{array}{l}\text { Universidad Nacional Mayor de } \\
\text { San Marcos }\end{array}$ & 4378 & 2471 & 1,77 \\
\hline 17 & $\begin{array}{l}\text { Universidad Andina Néstor Cáceres } \\
\text { Velásquez }\end{array}$ & 7 & 4 & 1,75 \\
\hline 18 & $\begin{array}{l}\text { Universidad Antonio Ruiz de } \\
\text { Montoya }\end{array}$ & 7 & 4 & 1,75 \\
\hline 19 & $\begin{array}{l}\text { Universidad Nacional Toribio } \\
\text { Rodríguez de Mendoza de } \\
\text { Amazonas }\end{array}$ & 13 & 8 & 1,63 \\
\hline 20 & $\begin{array}{l}\text { Universidad Nacional Autónoma de } \\
\text { Chota }\end{array}$ & 8 & 5 & 1,60 \\
\hline 21 & Universidad Católica San Pablo & 187 & 121 & 1,55 \\
\hline 22 & Universidad Nacional de Ingeniería & 687 & 447 & 1,54 \\
\hline 23 & Universidad Ricardo Palma & 234 & 155 & 1,51 \\
\hline 24 & Universidad Peruana Unión & 31 & 21 & 1,48 \\
\hline 25 & Universidad Nacional de Ucayali & 22 & 15 & 1,47 \\
\hline
\end{tabular}




\begin{tabular}{|c|c|c|c|c|}
\hline 26 & Universidad Nacional de Piura & 122 & 84 & 1,45 \\
\hline 27 & $\begin{array}{l}\text { Universidad Nacional de la } \\
\text { Amazonía Peruana }\end{array}$ & 219 & 151 & 1,45 \\
\hline 28 & $\begin{array}{l}\text { Universidad Nacional de San } \\
\text { Antonio Abad del Cusco }\end{array}$ & 523 & 361 & 1,45 \\
\hline 29 & Universidad Privada de Tacna & 23 & 16 & 1,44 \\
\hline 30 & $\begin{array}{l}\text { Universidad Privada Antenor } \\
\text { Orrego }\end{array}$ & 160 & 112 & 1,43 \\
\hline 31 & Universidad de Lima & 125 & 88 & 1,42 \\
\hline 32 & Universidad Alas Peruanas & 31 & 22 & 1,41 \\
\hline 33 & $\begin{array}{l}\text { Universidad Nacional Santiago } \\
\text { Antúnez de Mayolo }\end{array}$ & 39 & 28 & 1,39 \\
\hline 34 & $\begin{array}{l}\text { Universidad Nacional Agraria La } \\
\text { Molina }\end{array}$ & 641 & 462 & 1,39 \\
\hline 35 & $\begin{array}{l}\text { Universidad Nacional Federico } \\
\text { Villarreal }\end{array}$ & 172 & 126 & 1,37 \\
\hline 36 & Universidad César Vallejo S.A.C. & 47 & 35 & 1,34 \\
\hline 37 & Universidad Andina del Cusco & 16 & 12 & 1,33 \\
\hline 38 & $\begin{array}{l}\text { Universidad Católica Sedes } \\
\text { Sapientiae }\end{array}$ & 16 & 12 & 1,33 \\
\hline 39 & Universidad Nacional de Cajamarca & 78 & 59 & 1,32 \\
\hline 40 & $\begin{array}{l}\text { Universidad de Ingeniería y } \\
\text { Tecnología }\end{array}$ & 89 & 68 & 1,31 \\
\hline 41 & Universidad Autónoma del Perú & 12 & 10 & 1,20 \\
\hline 42 & Universidad de Piura & 285 & 239 & 1,19 \\
\hline 43 & $\begin{array}{l}\text { Universidad Nacional Agraria de la } \\
\text { Selva }\end{array}$ & 48 & 41 & 1,17 \\
\hline 44 & Universidad Peruana Los Andes & 14 & 12 & 1,17 \\
\hline 45 & $\begin{array}{l}\text { Universidad Nacional Daniel } \\
\text { Alcides Carrión }\end{array}$ & 15 & 13 & 1,15 \\
\hline 46 & $\begin{array}{l}\text { Universidad Privada Norbert } \\
\text { Wiener }\end{array}$ & 15 & 13 & 1,15 \\
\hline 47 & $\begin{array}{l}\text { Universidad Nacional de San } \\
\text { Martín }\end{array}$ & 24 & 21 & 1,14 \\
\hline 48 & $\begin{array}{l}\text { Universidad Nacional Jorge } \\
\text { Basadre Grohmann }\end{array}$ & 30 & 27 & 1,11 \\
\hline 49 & $\begin{array}{l}\text { Universidad Nacional de San } \\
\text { Agustín de Arequipa }\end{array}$ & 475 & 429 & 1,11 \\
\hline 50 & $\begin{array}{l}\text { Universidad Nacional San Luis } \\
\text { Gonzaga }\end{array}$ & 94 & 85 & 1,11 \\
\hline 51 & $\begin{array}{l}\text { Universidad Privada San Juan } \\
\text { Bautista S.A.C. }(* 6) \\
\end{array}$ & 42 & 38 & 1,11 \\
\hline 52 & Universidad Nacional de Trujillo & 364 & 330 & 1,10 \\
\hline 53 & Universidad Nacional del Altiplano & 111 & 101 & 1,10 \\
\hline 54 & Universidad Nacional del Santa & 20 & 19 & 1,05 \\
\hline 55 & Universidad Señor de Sipán & 20 & 19 & 1,05 \\
\hline 56 & $\begin{array}{l}\text { Universidad Católica de Santa } \\
\text { María }\end{array}$ & 117 & 113 & 1,04 \\
\hline 57 & $\begin{array}{l}\text { Universidad Nacional Pedro Ruiz } \\
\text { Gallo }\end{array}$ & 98 & 95 & 1,03 \\
\hline 58 & $\begin{array}{l}\text { Universidad Nacional Intercultural } \\
\text { de la Amazonía }\end{array}$ & 11 & 11 & 1,00 \\
\hline 59 & Universidad Tecnológica del Perú & 27 & 27 & 1,00 \\
\hline 60 & $\begin{array}{l}\text { Universidad Nacional de San } \\
\text { Cristóbal de Huamanga }\end{array}$ & 41 & 44 & 0,93 \\
\hline 61 & $\begin{array}{l}\text { Universidad Peruana de Ciencias } \\
\text { Aplicadas S.A.C. }\end{array}$ & 875 & 953 & 0,92 \\
\hline 62 & $\begin{array}{l}\text { Universidad Nacional del Centro } \\
\text { del Perú }\end{array}$ & 56 & 73 & 0,77 \\
\hline 63 & Universidad Nacional del Callao & 16 & 21 & 0,76 \\
\hline 64 & $\begin{array}{l}\text { Universidad Nacional de } \\
\text { Huancavelica }\end{array}$ & 34 & 48 & 0,71 \\
\hline
\end{tabular}

\begin{tabular}{|c|l|c|c|c|}
\hline 65 & Universidad Nacional de Tumbes & 22 & 36 & 0,61 \\
\hline 66 & $\begin{array}{l}\text { Universidad Católica Santo Toribio } \\
\text { de Mogrovejo - }\end{array}$ & 46 & 76 & 0,61 \\
\hline
\end{tabular}

Through these results can be observed that small universities rise in position when we compare the results of Table I and Table II with the results of Table III and Table IV.

\section{Discussion and Conclusions}

From Table I and Table II, it can be seen that most universities have a growth in the number of publications from 2017 to 2018. This growth is mainly due to the increasing importance that has been given to research in Peru due to many factors, among them, the new University Law, the accreditation process, and the competitive funds to finance research projects. It can be seen that the universities with the highest growth are Universidad Nacional Jorge Basadre Grohmann with 400 percent growth and Universidad Peruana Unión with 350 percent growth. Likewise, it can be seen that there are universities that show a decrease, these being the universities Universidad Ricardo Palma with 86.21 percent of regression and Universidad Nacional de Tumbes with 85.71 percent of regression. One can see in Figure 6, all publication evolution from 2017 to 2018 for the Peruvian universities.

The fact of using the number of REGINA researchers as a reference to taking into account the size of the university responds to the fact that there are opinions that indicate that not all professors carry out research, while REGINA investigators are called to carry out research. However, it is also true that some professors are not REGINA and publish scientific articles, as well as students who have publications. In that sense, the use of the number of authors identified in SCOPUS could be the best option.

In Figure 7, the distribution of the best 20 universities can be observed according to the different parameters considered in this study.

Finally, one can conclude that when one not only take into account the number of published scientific articles but also the size of the university, the ranking changes drastically resulting in great surprises, mainly in small universities that invest in research

\section{References}

[1] L. Medic, and J. Cieza, "Design projects in the first three years of a computing program", III IEEE World Engineering Education Conference - EDUNINE 2019, March 2019, Lima - Peru.

[2] Einstein, Albert. Einstein on politics: His private thoughts and public stands on nationalism, Zionism, war, peace, and the bomb. Princeton University Press, 2013.

[3] Gonzalez, Avid Roman. "Investigación: Oportunidad de Desarrollo."; XIII Congreso Nacional de Computacion, Informatica y Sistemas-CONACIS 2010. 2010 .

[4] Lastra, Javier Ulises Solis. "Tax Incentive to Promote Research Development and Innovation in Peru." 2018 IEEE Sciences and Humanities International Research Conference (SHIRCON). IEEE, 2018.

[5] De-Moya-Anegón, Félix; Herrán-Páez, Estefanía; Bustos-González, Atilio; Corera-Álvarez, Elena; Tibaná-Herrera, Gerardo (2018). Ranking Iberoamericano de instituciones de educación superior. SIR Iber 2018. Barcelona, España: Ediciones Profesionales de la Información SL. ISBN: 978 840903911 1. https://doi.org/10.3145/sir-iber-2018.

[6] Ránking Universidades Perú | AméricaEconomía. (2017). Rankings.americaeconomia.com. Retrieved 26 January 2017, from http://rankings.americaeconomia.com/2016/universidadesperu/metodologia.

[7] SUNEDU, "Informe Bienal Sobre la Realidad Universitaria Peruana", First Edition, 2018. https://www.sunedu.gob.pe/informe-bienal-sobrerealidaduniversitaria/. 


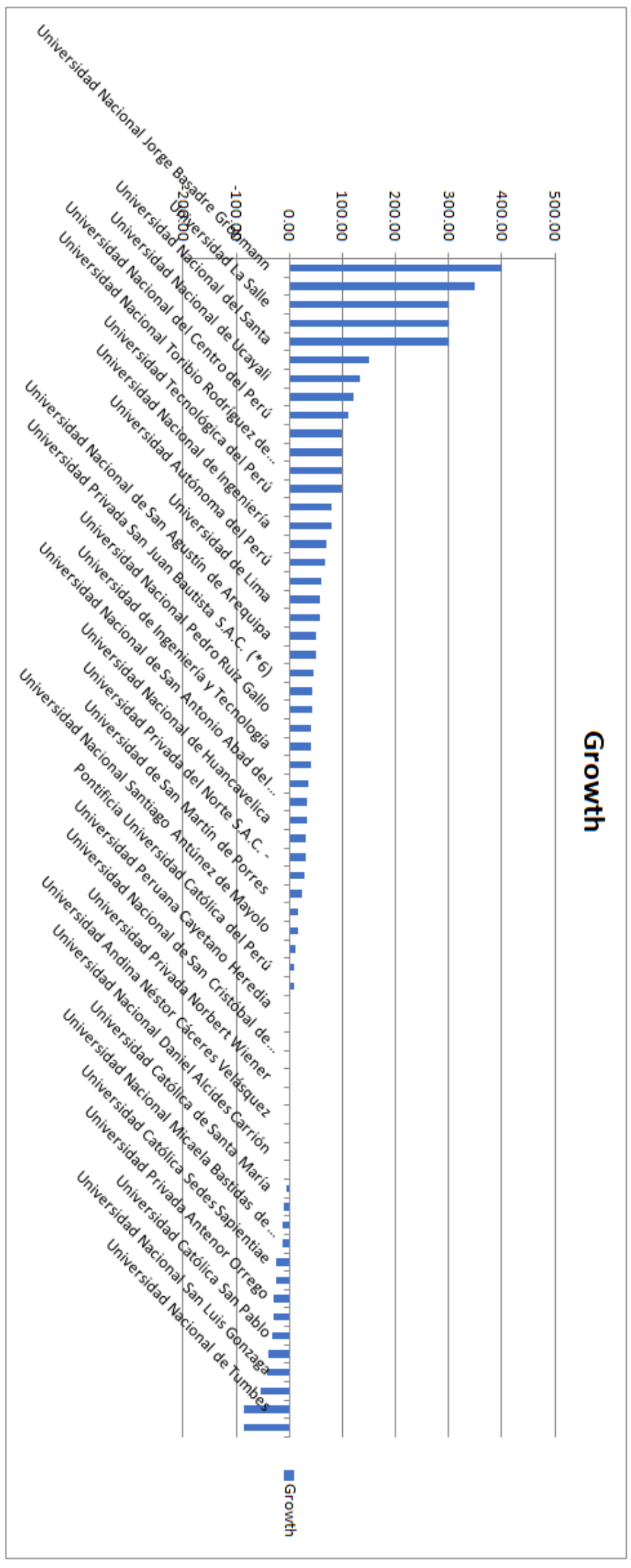

Figure 6: Distribution of the top 20 universities according to the number of publications and the ratios $R p / r$ and $R p / a$

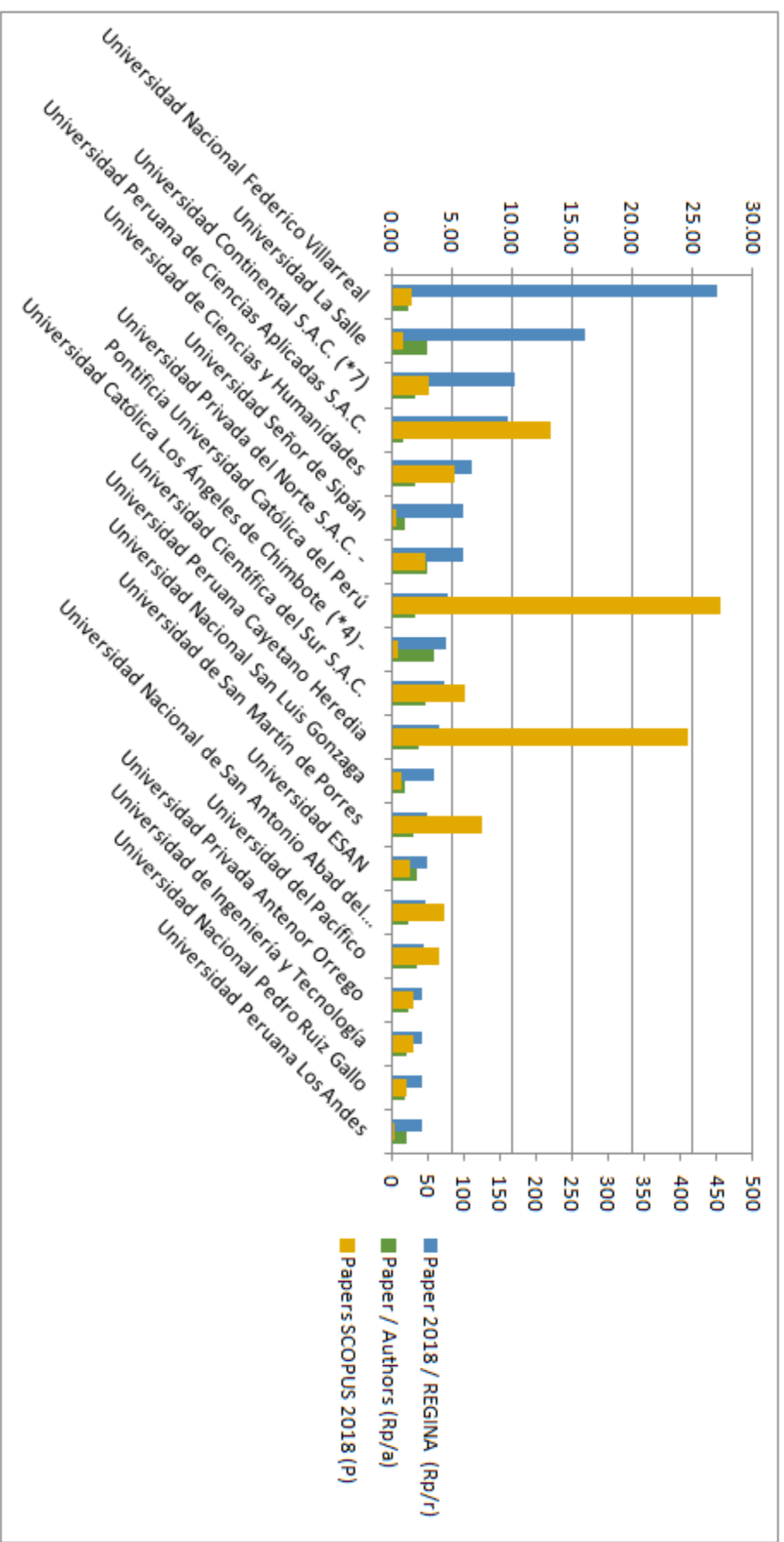

Figure 7: Distribution of the top 20 universities according to the number of publications and the ratios $R p / r$ and $R p / a$

[8] Docampo, D.; Herrera, F.; Luque-Martínez, T.; Torres-Salinas, D. (2012). Efecto de la agregación de universidades españolas en el Ranking de Shanghai (ARWU): caso de las comunidades autónomas y los campus de excelencia. El Profesional de la Información, 21 (4), 428-432. https://doi.org/10.3145/epi.2012.jul.16.

[9] Webometrics, "Ranking de Universidades", Edition 2018 2.1.2, http://www.webometrics.info/.

[10] QS, "Top Universities", https://www.topuniversities.com/qsworlduniversity-rankings.

[11] SIR Methodology. (2017). Scimagoir.com. Retrieved 26 January 2017, from $\mathrm{http}: / /$ www.scimagoir.com/methodology.php.

[12] Ahmad, Mohammad Esmail, et al. "The Role of Information Technology on Teaching Process in Education; An Analytical Prospective Study at University of Sulaimani." INTERNATIONAL JOURNAL OF ADVANCED COMPUTER SCIENCE AND APPLICATIONS 9.11 (2018): 512-521. 
[13] U. Lapa-Asto, G. Tirado-Mendoza, and A. Roman-Gonzalez, "Impact of Formative Research on Engineering Students", III IEEE World Engineering Education Conference - EDUNINE 2019, March 2019, Lima - Peru.

[14] Roman-Gonzalez, Avid, and Natalia I. Vargas-Cuentas. "Scientific Production in the 50 First Universities Licensed by SUNEDU." 2018 IEEE Sciences and Humanities International Research Conference (SHIRCON). IEEE, 2018.

[15] Mayta-Tristán, Percy, et al. "Producción científica y licenciamiento de escuelas de medicina en el Perú." Revista Peruana de Medicina Experimental y Salud Pública 36.1 (2019): 106-15.

[16] Estrada-Cuzcano, Alonso, and Karen Lizeth Alfaro-Mendives. "A propósito de REGINA: los investigadores de la Facultad de Letras y Ciencias Humanas." (2017).

[17] SUNEDU. "Informe Bienal Sobre la realidad Universitaria Peruana", Impresión Arte Peru SAC, Primera Edicion, 2018 